\title{
Collagen-induced arthritis
}

\author{
David D Brand ${ }^{1,2}$, Kary A Latham ${ }^{1,2}$, \& Edward F Rosloniec ${ }^{1,2}$
}

\begin{abstract}
${ }^{1}$ Research Service, Veterans Affairs Medical Center, Memphis, Tennessee 38104, USA. ${ }^{2}$ Departments of Medicine, Pathology and Molecular Sciences, University of Tennessee Health Science Center, Memphis, Tennessee 38163, USA. Correspondence should be addressed to E.F.R. (erosloniec@utmem.edu) or D.D.B. (dbrand@utmem.edu).

Published online 17 May 2007; doi:10.1038/nprot.2007.173

The collagen-induced arthritis (CIA) mouse model is the most commonly studied autoimmune model of rheumatoid arthritis. Autoimmune arthritis is induced in this model by immunization with an emulsion of complete Freund's adjuvant and type II collagen (CII). This protocol describes the steps necessary for acquisition, handling and preparation of CII, as well as selection of mouse strains, proper immunization technique and evaluation of the arthritis incidence and severity. Typically, the first signs of arthritis appear in this model 21-28 days after immunization, and identification of the arthritic limbs is not difficult. Using the protocol described, the investigator should be able to reproducibly induce a high incidence of CIA in various strains of genetically susceptible mice as well as learn how to critically evaluate the pathology of the disease. The total time for the preparation of reagents and the immunization of ten mice is about $1.5 \mathrm{~h}$.
\end{abstract}

\section{INTRODUCTION}

Animal models of autoimmune arthritis have proven to be valuable research tools for the study of pathogenic mechanisms of this disease as well as for testing new therapies. Several mouse models of arthritis have been established ${ }^{1}$, including those that require immunization with antigen-proteoglycan-induced arthritis $(\mathrm{PGIA})^{2}$, streptococcal cell-wall arthritis ${ }^{3}, \mathrm{CIA}^{4}$ and antigeninduced arthritis ${ }^{5}$; those induced by chemical agents-oil-induced arthritis ${ }^{6}$; and spontaneous models-tumor necrosis factor- $\alpha$ transgenic mouse ${ }^{7}$ and $\mathrm{K} / \mathrm{BxN}$ T-cell receptor transgenic mouse ${ }^{8}$. While each of these models has advantages and disadvantages, CIA has been the most widely studied model of rheumatoid arthritis (RA). It shares several pathological features with RA, and CII is a major protein in cartilage, the target tissue of RA. Additionally, of the antigen-defined models that are based on cartilage proteins, it has the shortest duration between immunization and disease manifestation. The CIA model has been used extensively to identify potential pathogenic mechanisms of autoimmunity, including the role of individual cell types in disease onset and progression, as well as to design and test new therapeutics. In recent years, the CIA model has been instrumental in the testing and development of the new biologically based therapeutics, such as those that target tumor necrosis factor- $\alpha$, a cytokine produced by macrophages and T cells that is a dominant inflammatory mediator in the pathogenesis of RA. The development of these biologically based therapies has revolutionized the treatment of RA.

CIA is elicited in genetically susceptible strains of mice by immunization with CII emulsified in complete Freund's adjuvant (CFA). The ensuing pathogenesis shares several pathological features with RA, including synovial hyperplasia, mononuclear cell infiltration, cartilage degradation, and, like RA, susceptibility is linked to the expression of specific MHC class II genes. The most notable differences between this model and RA are that rheumatoid factor is not present in CIA, there is little or no sex bias in CIA and that the experimental disease is generally monophasic, although some relapsing mouse models of CIA have been described ${ }^{9,10}$. While the presence of T-cell and B-cell immunity to CII has been reported in $\mathrm{RA}^{11-15}$, it is not clear if this is a causative factor or a result of the pathogenesis associated with this disease. The original "gold standard" of the CIA model was the DBA/1 (H-2q) mouse strain; however, in recent years, several HLA-DR mouse models have been established in which transgenic expression of the HLADR1 or DR4 class II genes associated with susceptibility to RA confers susceptibility to CIA in the recipient mouse strain ${ }^{16,17}$. These data indicate that the DR molecules associated with susceptibility to RA are at least involved in the immune response to CII.

The immunopathogenesis of CIA involves both a T-cell and B-cell-specific response to CII. The immunodominant T-cell determinants of CII that mediate CIA have been identified for most of the class II molecules that are associated with susceptibility to this experimental disease ${ }^{16-20}$, and a few have been studied in detail for their interaction with the class II molecule and T-cell receptor $^{21,22}$. Similarly, B-cell determinants targeted by the antibody response to CII have also been identified ${ }^{23,24}$, and there is some evidence that antibodies from RA patients target the same areas of the CII molecule as those from $\mathrm{CIA}^{25}$. Identification of pathogenic B-cell determinants has proven to be more difficult owing to the requirement that the pathogenic antibodies must be able to bind to the triple helical native CII. Unlike other autoimmune models such as EAE, where $\mathrm{T}$ cells are the primary pathogenic mechanism, the pathogenesis of CIA is mediated, in a large part, by CII-specific antibody that binds to the cartilage and is capable of fixing complement. Collectively, these data have enabled researchers to study a wide range of pathogenic mechanisms in this model, as well as to design and test novel therapeutics.

Despite the utility of the CIA mouse models, it is frequently difficult for the uninitiated investigator to establish this model in the laboratory and to develop the expertise to evaluate the associated pathology. In the protocol described below, we provide detailed descriptions and caveats for the preparation of CII for immunization, the method of immunization, a guide for strain selection and the most frequently used methods of evaluating the incidence and severity of the autoimmune arthritis. Disease incidences of $80-100 \%$ can be easily achieved for most of the susceptible strains, and identification of an arthritic limb is not difficult even with little experience. 


\section{MATERIALS}

\section{REAGENTS}

- Arlacel A (mannide monooleate; Fisher, cat. no. AC33213-0250), for laboratory prepared Freund's adjuvant

- Heavy paraffin (mineral) oil (Fisher, cat. no. 0122-1), for laboratory prepared Freund's adjuvant

- Heat-killed Mycobacterium tuberculosis strain H37Ra (BD Biosciences, cat. no. 231141), for laboratory prepared Freund's adjuvant

- Complete Freund adjuvant (BD Biosciences, cat. no. 231131), for commercially prepared Freund's adjuvant

- Incomplete Freund adjuvant (IFA; BD Biosciences, cat. no. 263910), for commercially prepared Freund's adjuvant

- Heat-killed M. tuberculosis strain H37Ra (BD Biosciences, cat. no. 231141), for commercially prepared Freund's adjuvant

- Type II collagen (Chondrex; MD Biosciences); see REAGENT SETUP

- Mice, see Table $\mathbf{1} \Delta$ CRITICAL See Table 1 for strain susceptibility information I CAUTION Experiments involving live rodents must conform to national and local regulations.

EQUIPMENT

- Mortar and pestle

- 3 or $5 \mathrm{ml}$ syringe (Becton Dickinson)

- Homogenizer (IKA-Werke Model \#T8.10)

- 18 gauge $\times 12$ needle (Becton Dickinson \#305196)

- 26 gauge $\times 12$ needle (Becton Dickinson \#305111)

\section{REAGENT SETUP}

Freund's adjuvant The best arthritis incidence is obtained if an emulsion is made with the proper adjuvant. Freund's adjuvant is not a complex mixture. It consists of $15 \%$ Arlacel A (v/v) in paraffin oil. Freund's adjuvant can be prepared in $100 \mathrm{ml}$ batches and stored sealed in rubber-stoppered glass Wheaton vials in $5 \mathrm{ml}$ aliquots. Adjuvants are very stable when stored at $4{ }^{\circ} \mathrm{C}$. Emulsions prepared with these adjuvants should have a consistency of dense whipped cream and should not disperse quickly when a droplet of emulsion is placed on the surface of a water-filled beaker. Commercially prepared Freund's adjuvant (complete and incomplete) can also be purchased from a number of vendors. CIA has been successfully induced in mice using these adjuvants, although most investigators supplement the complete adjuvant with 1-3 $\mathrm{mg}$ per $\mathrm{ml}$ of $M$. tuberculosis. $\Delta$ CRITICAL For reasons unknown to the authors, during the mid-1990s, commercial suppliers of Freund's adjuvants switched to using light paraffin oils instead of the heavy oils that were commonly used before that time. The Freund's adjuvants that are prepared with light mineral oils have a lower viscosity, and the emulsions made with these adjuvants make lower-quality emulsions compared to those made with heavy mineral oil. Until such time as adjuvant vendors resume preparing adjuvants with heavy paraffin oil, it may be advisable to prepare your own using the simple methods outlined below.
Complete Freund's adjuvant Most mouse strains require immunization with CFA for elicitation of a high incidence of arthritis. This is likely due to the stimulatory capacity of heat-killed Mycobacterium in the adjuvant and its promotion of the inflammatory response that initiates an immune response to CII. This concept is supported by the fact that a higher than usual concentration of the heat-killed M. tuberculosis strain H37Ra is required to elicit a high incidence of disease in the CIA model (2-4 mg ml $\mathrm{m}^{-1}$ in the adjuvant in comparison to the commercially available $1 \mathrm{mg} \mathrm{ml}^{-1}$ ).

! CAUTION Institutional Animal Care and Use Committees (IACUCs) are very cautious about the use of CFA in rodents. AVMA guidelines supplied to the IACUCs suggest that adjuvants other than CFA can be successfully used in the generation of antibodies. While this is true, most IACUCs have little or no experience in CIA disease induction, and do not understand the important role played by $M$. tuberculosis $\mathrm{H} 37 \mathrm{Ra}$ in the generation of the autoimmune response that drives the pathogenesis of this disease process. The guidelines to which most IACUCs adhere were designed strictly to provide a framework for the generation of an antibody-induction-driven immune response and not a complex disease process such as CIA. However, followed carefully, this protocol can help the investigator to avoid the elicitation of tail ulcers or orchitis that can result from improperly applied emulsion, and will provide a timetested method for the successful induction of arthritis in rodents. Use of lower quantities of M. tuberculosis as suggested by IACUCs for production of antibody can result in lower CIA disease incidence.

Collagen preparation Collagen is a fibrous protein that, at normal physiological conditions, is insoluble. Consequently, to keep CII in solution for use in the CIA model, several precautions need to be observed. CII must be solubilized and stored in a dilute solution of acetic acid. Concentrations of 10 and $100 \mathrm{mM}$ acetic acid can be used, but for immunization purposes, $10 \mathrm{mM}$ is preferred to reduce deleterious effects to the mouse. If soluble $\mathrm{CII}$ comes into contact with salts (such as $\mathrm{NaCl}$, and especially PBS), it may precipitate. When prepared fresh, CII is a clear, colorless solution. Over time (even when frozen), CII solubility will decrease, causing some precipitation of the protein. If this occurs, the solution will become cloudy. Centrifugation at $10,000 \mathrm{~g}$ for $30 \mathrm{~min}$ at $4{ }^{\circ} \mathrm{C}$ will remove the insoluble material; however, the concentration of the soluble $\mathrm{CII}$ in the supernatant cannot be accurately determined without relyophilization and weighing the protein. If CII is lyophilized (or if purchased in lyophilized form), it should be dissolved in dilute acetic acid to the desired concentration $\left(2-4 \mathrm{mg} \mathrm{ml}^{-1}\right)$ by gentle stirring for several hours to overnight. This should be carried out at $4{ }^{\circ} \mathrm{C}$ using reagents and glassware that are prechilled to avoid denaturing CII. Proper care should be taken to ensure that the stir plate on which CII is being mixed does not generate heat even when in the cold room. Placing one's hand on the surface of a stir platform that has been operating for $30 \mathrm{~min}$ in the cold will allow one to determine if it is safe to use. Once the collagen has been dissolved to its desired concentration, it should be placed in aliquots and frozen at $-70{ }^{\circ} \mathrm{C}$ so that it does not remain thawed at $4{ }^{\circ} \mathrm{C}$ for more than $1-2$ months.

\section{PROCEDURE}

\section{Preparation of IFA TIMING 10 min}

1| Prepare a mixture of Arlacel A and mineral oil composed of $15 \%$ Arlacel A and $85 \%$ mineral oil.

PAUSE POINT This mixture can be made in large batches and then aliquoted and stored at $4{ }^{\circ} \mathrm{C}$ for later use.

\section{Preparation of CFA $\bigcirc$ TIMING 20 min}

2| Calculate the amount of heat-killed M. tuberculosis required to make a $4 \mathrm{mg} \mathrm{ml}^{-1}$ solution of $M$. tuberculosis in IFA.

3| Grind M. tuberculosis to a fine powder using a mortar and pestle.

4| Weigh the resulting M. tuberculosis powder and add the appropriate amount to IFA.

$\triangle$ CRITICAL STEP The easiest way to transfer ground

M. tuberculosis to IFA is to grind slightly more M. tuberculosis than is required and then to weigh the fine powder before its addition to IFA. The alternative approach, weighing the exact amount required before grinding and then attempting to transfer all of the ground powder directly from the mortar to the container holding IFA, is relatively imprecise and difficult.
TABLE 1 | Strains of mice susceptible to CIA.

\begin{tabular}{llll}
\hline $\begin{array}{l}\text { Susceptible } \\
\text { strains }\end{array}$ & $\begin{array}{l}\text { Class II } \\
\text { haplotype }\end{array}$ & $\begin{array}{l}\text { Arthritis } \\
\text { incidence (\%) }\end{array}$ & $\begin{array}{l}\text { Arthritis } \\
\text { onset }\end{array}$ \\
\hline DBA/1 & I-A $^{q}$ & $80-100$ & Days 21-28 \\
B10.M-DR1 & DRB1*0101 $^{*}$ & $80-100$ & Days 21-28 \\
B10.M-DR4 & DRB1*0401 $^{*}$ & $50-80^{\mathrm{a}}$ & Days 21-28 \\
B10.RIII & I-A $^{\mathrm{r}}$ & $80-100$ & Days 21-28 \\
B10.0 & I-A $^{q}$ & $80-100$ & Days 21-28 \\
DBA-TCR Tg & I-A $^{q}$ & $80-100$ & Days 12-18 \\
C57BL/6 & I-A $^{\mathrm{b}}$ & Variable $^{\mathrm{b}}$ & Variable $^{\mathrm{b}}$ \\
\hline
\end{tabular}

The lower incidence of arthritis in this strain is likely a result of the fact that this particular transgenic strain must be bred as a heterozygous mouse ${ }^{17}$. ${ }^{b}$ There are conflicting data on the susceptibility of the B6 strain to CIA with some reporting little or no incidence ${ }^{28-30}$ and others reporting as high as $60 \%$ incidence ${ }^{31}$. 
5| Vortex CFA vigorously before use to thoroughly suspend $M$. tuberculosis in the Freund's adjuvant.

PAUSE POINT CFA can be stored for long periods of time at $4{ }^{\circ} \mathrm{C}$ without losing effectiveness, but will require mixing by vortex before each use.

\section{Preparation of emulsion $\bigcirc$ TIMING 20 min}

6| Calculate the volumes of collagen and CFA required to make the emulsion, keeping their ratios at 1:1. While $50 \mu \mathrm{l}$ of emulsion is required per mouse, extra must be made owing to both losses during processing and the dead space in the syringes. $\triangle$ CRITICAL STEP Using a tissue homogenizer (described below) to emulsify, the minimum final volume that can be emulsified is $0.5 \mathrm{ml}$. Additionally, regardless of the procedure used to emulsify, plan on at least a 200-300 $\mu$ l loss of material during preparation and transfer to the immunization syringe.

7| Select an appropriate container in which to mix the emulsion.

$\triangle$ CRITICAL STEP There are many different protocols for preparing emulsions. We have found that the most effective method is to mechanically mix the emulsion in a small syringe with a capacity of at least $1 \mathrm{ml}$ more than the emulsion volume using a tissue homogenizer (IKA-Werke Model \#T8.10). This allows both thorough emulsification of CFA and CII and easy transfer to a syringe appropriate for immunization. The primary advantage of this approach is a high-quality emulsification. The primary drawback is that all reagents and the emulsion must be kept cold during the procedure. Our protocol below reflects these methodological preferences.

8| Stopper the syringe in which the emulsion will be made and add the required volume of CFA directly to the syringe. $\triangle$ CRITICAL STEP Keep all reagents on ice as much as possible during emulsification. Keeping the reagents cold prevents denaturing of CII, which is paramount to eliciting a high incidence of disease in the mouse model.

9| Add the collagen drop by drop to CFA while the emulsion is mixed at medium speed. Once the collagen has been added, increase the speed of the homogenizer to maximum for approximately 2 min. This will produce heat, so it is important that the syringe containing the emulsion be cooled while emulsification is taking place. Placing the syringe in ice stabilizes the syringe during emulsification and also keeps it at the required low temperature.

10| Remove the emulsion from the homogenizer and check it visually to ensure that the emulsion appears to be thoroughly mixed and is of the appearance and viscosity of dense whipped cream. If the degree of emulsification is not satisfactory, further mixing with the homogenizer should improve the quality of the emulsion.

\section{Transfer of the emulsion to a $\mathbf{1} \mathbf{~ m l}$ syringe for immunization TIMING $\mathbf{5}$ min}

11 Using a large bore needle (18 gauge), carefully make a small hole in the syringe containing the emulsion at the top of the emulsion volume. This will allow release of air from the syringe, as the plunger is depressed during the transfer to the immunization syringe.

! CAUTION Great care should be observed so as not to inadvertently cause a needle stick, as CFA can induce a serious inflammatory response.

12| Connect the syringe in which the emulsion was made to the immunization syringe using a $1 \mathrm{~cm}$ length of plastic tubing. Depress the syringe plunger to slowly transfer the emulsion to the immunization syringe. The hole introduced in Step 1 above will allow the air to escape, improving the transfer.

13| Attach a $26 \times 1 / 2$ gauge needle to the immunization syringe and place on ice.

\section{Immunization $\bigcirc$ TIMING 2 min per mouse}

14| Although animals do not need to be anesthetized for immunization, it is significantly easier to inject the emulsion in the proper tissue layer while the mice are under anesthesia (isofluorane), and many IACUCs require this. Choose an injection site about $1.5 \mathrm{~cm}$ distal from the base of the tail, being careful to choose a tissue site and not a vessel. If a booster immunization will be given at a later time point (see Step 15), be sure to leave sufficient space above the immunization site for the booster injection. Slowly inject a $50 \mu$ volume intradermally (i.d.) into the tail (Fig. 1). There will be noticeable tissue resistance to the injection. A rapid, easy injection can be indicative of a subcutaneous or deep tissue injection and can result in a poor incidence of disease.

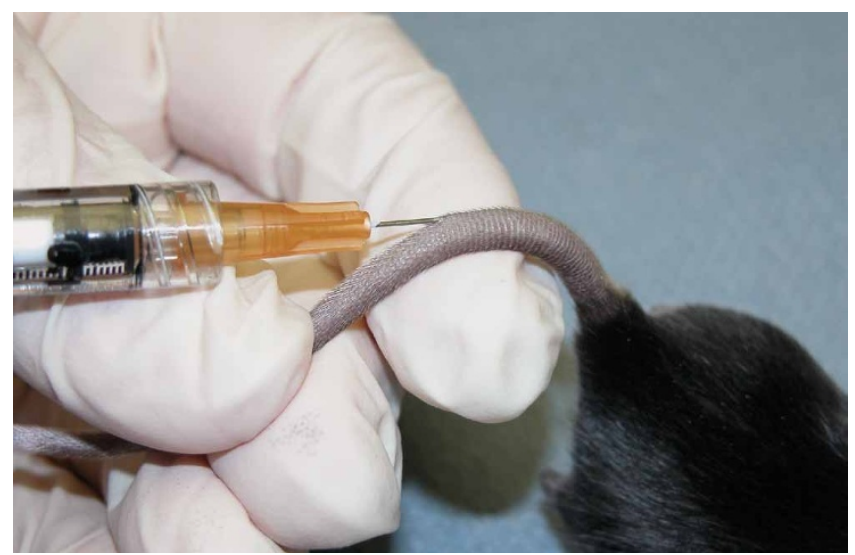

Figure 1 | Proper technique for immunization of mice for the induction of CIA. Emulsified CII in CFA is injected i.d. in the skin of the tail at approximately $1.5 \mathrm{~cm}$ distal to the base of the tail. 
15 While not all investigators find it necessary, a booster or secondary immunization is sometimes used 14 or 21 days after the primary immunization to ensure induction of a high incidence of CIA. The same concentration of CII is used as for the primary immunization; however, CII is emulsified in IFA for this immunization (see cautionary note below). The injection site is again i.d. in the tail, but proximal to the primary injection site. Care must be taken to avoid injecting near any of the inflamed tissue that may be present from the primary immunization. Booster immunizations will not correct problems that may have occurred with the primary immunization.

! CAUTION The use of CFA in the booster immunization is not allowed in most institutions. The investigator should consult their IACUC before considering this approach.
TABLE 2 | Scoring system for subjective evaluation of arthritis severity.

\begin{tabular}{ll}
\hline $\begin{array}{l}\text { Severity } \\
\text { score }\end{array}$ & Degree of inflammation \\
\hline 0 & $\begin{array}{l}\text { No evidence of erythema and swelling } \\
\text { Erythema and mild swelling confined to the tarsals or ankle } \\
\text { joint }\end{array}$ \\
2 & $\begin{array}{l}\text { Erythema and mild swelling extending from the ankle to } \\
\text { the tarsals } \\
3\end{array}$ \\
$\begin{array}{l}\text { Erythema and moderate swelling extending from the ankle } \\
\text { to metatarsal joints } \\
\text { Erythema and severe swelling encompass the ankle, foot } \\
\text { and digits, or ankylosis of the limb }\end{array}$ \\
\hline
\end{tabular}

\section{Monitoring of arthritis incidence TIMING 5-8 weeks}

16 Animals should be evaluated two to three times per week for arthritis incidence (as discussed in ANTICIPATED RESULTS). Each paw should be evaluated and scored individually on a scale of $0-4$, with 4 indicating the most severe inflammation (see Table 2). ? TROUBLESHOOTING

\section{TIMING}

IFA preparation: $10 \mathrm{~min}$ (Step 1)

CFA preparation: 20 min (Steps 2-5)

Emulsification of collagen and CFA: 20 min (Steps 6-10)

Transfer of emulsion to injection syringe: 5 min (Steps 11-13)

Immunization: 2 min per animal (Step 14)

Monitoring of arthritis incidence: $5-8$ weeks (Step 15)

\section{? TROUBLESHOOTING}

Troubleshooting advice can be found in Table 3.

TABLE 3 | Troubleshooting table.

\begin{tabular}{|c|c|c|}
\hline Problem & Possible cause & Solution \\
\hline \multirow[t]{6}{*}{$\begin{array}{l}\text { Low incidence/ } \\
\text { no arthritis }\end{array}$} & Denatured CII & $\begin{array}{l}\text { Keep the collagen cold, do not store at } 4{ }^{\circ} \mathrm{C} \text { for more than } 2 \text { months. Consider storing the } \\
\text { collagen in small aliquots so that experimental quantities can be thawed when needed. } \\
\text { Collagen should be a clear solution. Cloudy collagen suggests that it has crosslinked and } \\
\text { precipitated, or that it may be contaminated and should be discarded }\end{array}$ \\
\hline & Poor emulsion & (see "unstable emulsion" as a problem below) \\
\hline & $\begin{array}{l}\text { Inadequate concentration } \\
\text { of } M \text {. tuberculosis }(M t b)\end{array}$ & $\begin{array}{l}\text { Did IFA get used by mistake? Consider using freshly ground } M t b \text { strain } \mathrm{H} 37 \mathrm{Ra} \text {, or increase } \\
\text { the concentration of Mtb in the CFA. Make sure Mtb is finely ground. Make certain that the } \\
\text { Mtb in the Freund's adjuvant is vortexed sufficiently to resuspend it before use }\end{array}$ \\
\hline & Subcutaneous injection & $\begin{array}{l}\text { Make sure to keep the injection shallow to maintain intradermal site. Avoid injections } \\
\text { that go deep into the tail tissue }\end{array}$ \\
\hline & Aged or immature mice & Mice at $8-12$ weeks of age are optimum for starting experiments \\
\hline & Health status of mice & Are the mice healthy at the beginning of the experiment? Check for proper grooming \\
\hline
\end{tabular}

Unstable emulsion Insufficient mixing Thermal stability Poor mixing technique

Orchitis Injection sequelae

Ulceration/loss of fur Injection sequelae

Mouse dies
Injection sequelae Anesthesia sequelae
Increase the speed of the mixing apparatus (homogenizer, dual-hub syringe, etc.)

Keep the reagents cold and the mixing vessel on ice during emulsification

Start with oil and add aqueous solution slowly and dropwise while mixing

Emulsion has entered the urogenital region in mouse. Move the injection site distally on the mouse tail

Emulsion should stay in the intradermal region on the tail, and not move to the body of the mouse

Be cautious not to inject emulsion into the tail vein, as this can be fatal Monitor anesthesia carefully during procedure 


\section{ANTICIPATED RESULTS}

The intradermal immunization of mice in the tail with CFA and CII results in a moderate inflammatory reaction at the injection site that lasts 1-2 weeks. For most of the CIA-susceptible strains of mice, the first signs of arthritis development are visible between days 18 and 25 after immunization. The incidence of arthritis in CIA-susceptible strains of mice is generally very high (Table 1 ), reaching $80-100 \%$ in most strains, and is very consistent from experiment to experiment when high-quality emulsions are used and the immunization technique is mastered. However, the onset of disease for each mouse in the same experimental group can vary widely. As demonstrated in Figure 2 with the $\mathrm{DBA} / 1$ mouse strain, arthritis develops over a wide range of time, with peak incidence reached anywhere from 40 to 50 days after immunization. Additionally, the kinetics of arthritis development can vary from experiment to experiment as evidenced by the comparison of the incidence rate in the two independent experiments shown in Figure 2. Older male DBA/1 mice ( $>4$ months of age) should be avoided for CIA experiments as there is some evidence that these mice develop a spontaneous arthritis ${ }^{26,27}$. These patterns are similar in all of the arthritis-susceptible strains, with the possible exception of the C57BL/6 (B6) mouse. The susceptibility of B6 mice to CIA is presently controversial. Some investigators have reported little or no incidence of CIA in these mice ${ }^{28-30}$ while others have clearly demonstrated that CIA can be established in this strain ${ }^{31,32}$. At least some of this variability appears to be related to the vendor source of the mice, although a number of other factors including the species of CII and quantity of Mycobacterium used in the adjuvant may also be involved. Regardless, establishment of the CIA model in B6 mice has significant advantages due to the availability of numerous B6 knockout strains.

Visual identification of an arthritic limb is not difficult, with the possible exception of very early and mild disease. Several examples of arthritic hindlimbs and forelimbs are shown in Figure 3. For each of these limbs, arthritis is clearly evident although variations in degree or severity of the inflammation are present. Quantifying the severity of the arthritis in each limb can be problematic. Commonly, a subjective scoring system is applied to each limb using a scale of $0-4$, with 4 being the most severe inflammation (Table 2), and this includes ankylosis of the limb, arguably the severest form of arthritis. While useful, the subjectivity of this approach can be a concern for quantitative analysis of disease severity. Some investigators have used calipers to measure ankle or foot thickness; however, this approach does not allow incorporation of data involving changes throughout the affected limb and relies heavily on precise, reproducible placement of the calipers from mouse to mouse. An attractive alternative is the use of a plethysmometer, a microprocesor-controlled volume meter for measuring paw volume (Ugo Basile). By dipping the paw in a liquid-filled cell, the volume of the paw is calculated by this instrument based on the displacement of the liquid. Although this is a more accurate and reproducible approach that analyzes the entire paw, it can be very time consuming for large-scale experiments. Regardless of the approach used to evaluate arthritis severity, there are a number of ways to analyze the severity data, including score per limb, sum of the scores per mouse, as well as numbers of arthritic limbs per mouse or per experimental group. Two examples of these data analyses are shown in Figure 4, where the mean number of arthritic limbs per arthritic mouse, and the mean score per arthritic limb were calculated. These analyses are especially useful when arthritis incidence is similar between groups and the basis of the experimental design was to determine the efficacy of a therapy in reducing inflammation. However, when two groups vary significantly in their incidence of disease, analysis of severity data as a per group measurement (arthritic and nonharthritic mice) should be avoided, as it will only serve to recapitulate the incidence data. For most analyses, severity expressed as a function of either the number of arthritic mice or number of arthritic limbs serves as the best measurement; however, the best approach for analysis of these data should be based on the scientific questions being addressed.
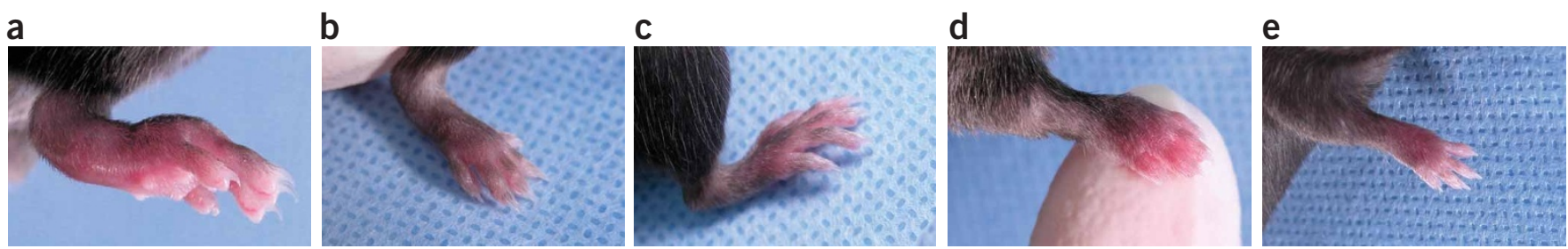

Figure 3 | Arthritic hindlimbs and forelimbs in mice immunized with CII emulsified in CFA. (a-c) Arthritic hindlimbs with severity scores of 4 (a), 3 (b) and 2 (c). Pictures clearly demonstrate how the inflammation progresses up the limb as the severity of the arthritis increases. (d, e) Arthritic forelimbs with severity scores of $4(\mathbf{d})$ and $3(\mathbf{e})$. 
For identification and evaluation of arthritic limbs, it is good practice to examine each limb of each mouse 2-3 times per week, as not only will the onset vary among mice in the same experimental group, but the appearance of arthritis in each limb will vary for each mouse. Within an individual mouse, arthritis can occur in any limb or combination of limbs at any time, and each limb of an individual mouse can develop arthritis at a different time point. There is some evidence that arthritis develops earliest and is more common in the hind limbs, but exceptions to this rule are often observed. Why arthritis develops in one limb of a mouse and not the other is unclear.

Finally, in addition to the severity and incidence of arthritis, the autoimmune response to CII can also be evaluated by measuring CII-specific T-cell proliferative responses in vitro ${ }^{16,20}$ or by measuring the quantity of the CII-specific antibody in the sera of the mice ${ }^{24}$. Both these measurements relate well to the development of autoimmunity, and the quantity of CII-specific antibody correlates with the presence or absence of arthritis.

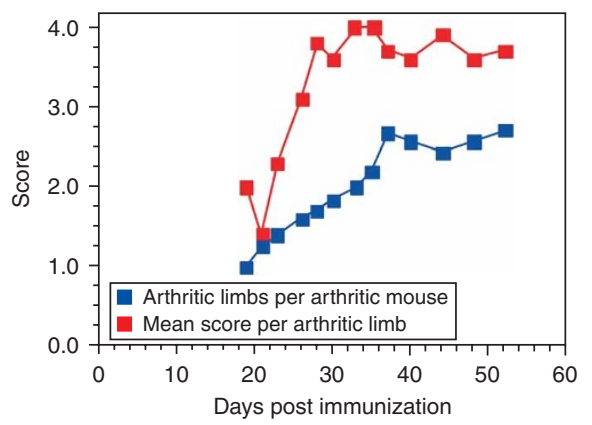

Figure 4 | Severity scores of arthritic limbs from DBA/1 mice immunized with CII emulsified in CFA. The number of arthritic limbs were quantitated and each limb assigned a severity score of 0-4 according to the criteria in Table 2. Data indicate the number of arthritic limbs per arthritic mouse (blue token) and the mean severity score of each arthritic limb (red token).
COMPETING INTERESTS STATEMENT The authors declare that they have no competing financial interests.

Published online at http://www.natureprotocols.com

Rights and permissions information is available online at http://npg.nature.com/ reprintsandpermissions

1. Brand, D.D. Rodent models of rheumatoid arthritis. Comp. Med. 55, 114-122 (2005).

2. Finnegan, A., Mikecz, K., Tao, P. \& Glant, T.T. Proteoglycan (aggrecan)-induced arthritis in BALB/C mice is a Th1-type disease regulated by Th2 cytokines. J. Immunol. 163, 5383-5390 (1999).

3. Koga, T. et al. Acute joint inflammation in mice after systemic injection of the cell wall, its peptidoglycan, and chemically defined peptidoglycan subunits from various bacteria. Infect. Immun. 50, 27-34 (1985).

4. Courtenay, J.S., Dallman, M.J., Dayan, A.D., Martin, A. \& Mosedale, B. Immunization against heterologous type II collagen induces arthritis in mice. Nature 283, 666-668 (1980).

5. Brackertz, D., Mitchell, G.F. \& Mackay, I.R. Antigen-induced arthritis in mice. I. Induction of arthritis in various strains of mice. Arthritis Rheum. 20, 841-850 (1977).

6. Hopkins, S.J., Freemont, A.J. \& Jayson, M.I. Pristane-induced arthritis in Balb/c mice. I. Clinical and histological features of the arthropathy. Rheumatol. Int. 5, 21-28 (1984).

7. Butler, D.M. et al. DBA/1 mice expressing the human TNF-alpha transgene develop a severe, erosive arthritis: characterization of the cytokine cascade and cellular composition. J. Immunol. 159, 2867-2876 (1997).

8. Kouskoff, V. et al. Organ-specific disease provoked by systemic autoimmunity. Cell 87, 811-822 (1996).

9. Holmdahl, R., Jansson, L., Larsson, E., Rubin, K. \& Klareskog, L. Homologous type II collagen induces chronic and progressive arthritis in mice. Arthritis Rheum. 29, 106-113 (1985).

10. Boissier, M.C., Feng, X.Z., Carlioz, A., Roudier, R. \& Fournier, C. Experimental autoimmune arthritis in mice. I. Homologous type II collagen is responsible for self-perpetuating chronic polyarthritis. Ann. Rheum. Dis. 46, 691-700 (1987).

11. Londei, M. et al. Persistence of collagen type II-specific T-cell clones in the synovial membrane of a patient with rheumatoid arthritis. Proc. Natl. Acad. Sci. USA 86, 636-640 (1989).

12. Kim, H.Y. et al. Enhanced T cell proliferative response to type II collagen and synthetic peptide CII (255-274) in patients with rheumatoid arthritis. Arthritis Rheum. 42, 2085-2093 (1999).

13. Terato, K. et al. Specificity of antibodies to type II collagen in rheumatoid arthritis. Arthritis Rheum. 33, 1493-1500 (1990).

14. Kim, W.U. et al. IgG antibodies to type II collagen reflect inflammatory activity in patients with rheumatoid arthritis. J. Rheumatol. 27, 575-581 (2000).

15. Watson, W.C., Tooms, R.E., Carnesale, P.G. \& Dutkowsky, J.P. A case of germinal center formation by CD45RO T and CD20 B lymphocytes in rheumatoid arthritic 
29. Chu, C.Q., Song, Z., Mayton, L., Wu, B. \& Wooley, P.H. IFNgamma deficient C57BL/ $6(\mathrm{H}-2 \mathrm{~b})$ mice develop collagen induced arthritis with predominant usage of T cell receptor Vbeta6 and Vbeta8 in arthritic joints. Ann. Rheum. Dis. 62, 983-990 (2003).

30. Pan, M., Kang, I., Craft, J. \& Yin, Z. Resistance to development of collageninduced arthritis in $\mathrm{C} 57 \mathrm{BL} / 6$ mice is due to a defect in secondary, but not in primary, immune response. J. Clin. Immunol. 24, 481-491 (2004).
31. Campbell, I.K., Hamilton, J.A. \& Wicks, I.P. Collagen-induced arthritis in $\mathrm{C} 57 \mathrm{BL} / 6(\mathrm{H}-2 \mathrm{~b})$ mice: new insights into an important disease model of rheumatoid arthritis. Eur. J. Immunol. 30, 1568-1575 (2000).

32. Kai, H. et al. Critical role of M. tuberculosis for dendritic cell maturation to induce collagen-induced arthritis in $\mathrm{H}-2 \mathrm{~b}$ background of $\mathrm{C} 57 \mathrm{BL} / 6$ mice. Immunology 118, 233-239 (2006). 